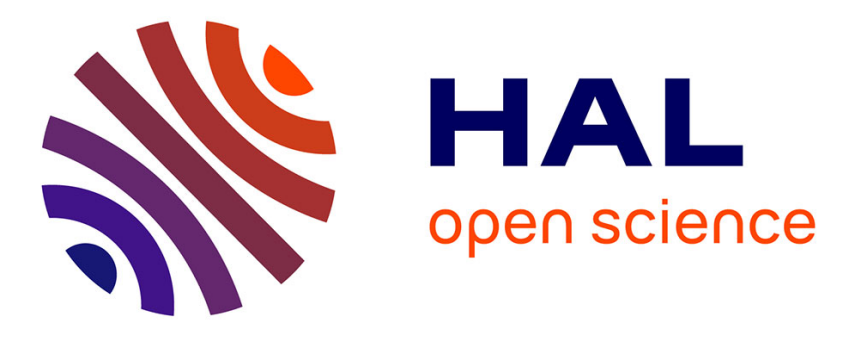

\title{
Zinc Induces Temperature-Dependent Reversible Self-Assembly of Tau
}

Andrei Yu Roman, François Devred, Deborah Byrne, Romain La Rocca, Natalia N Ninkina, Vincent Peyrot, Philipp O Tsvetkov, Andrei Yu. Roman

\section{- To cite this version:}

Andrei Yu Roman, François Devred, Deborah Byrne, Romain La Rocca, Natalia N Ninkina, et al.. Zinc Induces Temperature-Dependent Reversible Self-Assembly of Tau. Journal of Molecular Biology, 2018, 10.1016/j.jmb.2018.12.008 . hal-01981683v2

\section{HAL Id: hal-01981683 \\ https: / hal-amu.archives-ouvertes.fr/hal-01981683v2}

Submitted on 5 Feb 2019

HAL is a multi-disciplinary open access archive for the deposit and dissemination of scientific research documents, whether they are published or not. The documents may come from teaching and research institutions in France or abroad, or from public or private research centers.
L'archive ouverte pluridisciplinaire HAL, est destinée au dépôt et à la diffusion de documents scientifiques de niveau recherche, publiés ou non, émanant des établissements d'enseignement et de recherche français ou étrangers, des laboratoires publics ou privés.

\section{(c)(1)}

Distributed under a Creative Commons Attribution| 4.0 International License 


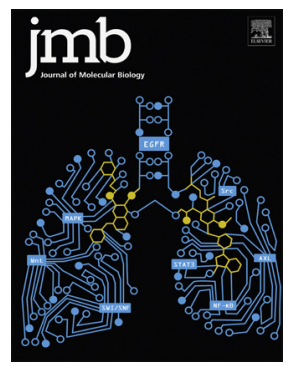

\title{
Zinc Induces Temperature-Dependent Reversible Self-Assembly of Tau
}

\author{
Andrei Yu. Roman ${ }^{1}$, François Devred ${ }^{2}$, Deborah Byrne ${ }^{3}$, Romain La Rocca ${ }^{2}$, \\ Natalia N. Ninkina ${ }^{4}$, Vincent Peyrot $^{2}$ and Philipp O. Tsvetkov ${ }^{2}$
}

1 - Aix-Marseille Univ, Inserm, CRO2 UMR_S 911, Faculté de Pharmacie, Marseille, France

2 - Aix-Marseille Univ, CNRS, INP, Inst Neurophysiopathol, Fac Pharm, Marseille, France

3 - Institut de Microbiologie de la Méditerranée, CNRS, FR3479, Aix-Marseille Univ, Marseille, France

4 - School of Biosciences, Cardiff University, Sir Martin Evans Building, Museum Avenue, Cardiff, CF10 3AX, UK

Correspondence to François Devred and Natalia N. Ninkina: andrei.roman@etu.univ-amu.fr, francois.devred@ univ-amu.fr, byrne@imm.cnrs.fr, ninkinan@cardiff.ac.uk,vincent.peyrot@univ-amu.fr, philipp.tsvetkov@univ-amu.fr https://doi.org/10.1016/j.jmb.2018.12.008

Edited by Louise C. Serpell

\begin{abstract}
Tau is an intrinsically disordered microtubule-associated protein that is implicated in several neurodegenerative disorders called tauopathies. In these diseases, Tau is found in the form of intracellular inclusions that consist of aggregated paired helical filaments (PHFs) in neurons. Given the importance of this irreversible PHF formation in neurodegenerative disease, Tau aggregation has been extensively studied. Several different factors, such as mutations or post translational modifications, have been shown to influence the formation of late-stage non-reversible Tau aggregates. It was recently shown that zinc ions accelerated heparin-induced oligomerization of Tau constructs. Indeed, in vitro studies of PHFs have usually been performed in the presence of additional co-factors, such as heparin, in order to accelerate their formation. Using turbidimetry, we investigated the impact of zinc ions on Tau in the absence of heparin and found that zinc is able to induce a temperature-dependent reversible oligomerization of Tau. The obtained oligomers were not amyloid-like and dissociated instantly following zinc chelation or a temperature decrease. Finally, a combination of isothermal titration calorimetry and dynamic light scattering experiments showed zinc binding to a high-affinity binding site and three low-affinity sites on Tau, accompanied by a change in Tau folding. Altogether, our findings stress the importance of zinc in Tau oligomerization. This newly identified Zn-induced oligomerization mechanism may be a part of a pathway different of and concurrent to Tau aggregation cascade leading to PHF formation.
\end{abstract}

(C) 2018 Published by Elsevier Ltd.

\section{Introduction}

Microtubule (MT)-associated protein Tau is known to play a vital role in cytoskeleton regulation by binding tubulin and thus controlling essential MT functions, from the necessary dynamic instability in dividing cells to axonal stability in neurons $[1,2]$. Tau is also associated with a number of neurodegenerative diseases (NDs) including Alzheimer's disease $(A D)$, Parkinson's disease (PD), Pick's disease, frontotemporal lobar degeneration, corticobasal degeneration and progressive supranuclear palsy, where it forms intracellular aggregates [3-6]. These aggregates would spread in the brain through prion-like propagation [3,7]. In the case of $A D$, Tau is believed to be the main factor underlying the development and progression of the pathology [8]. Different types of inclusions made of abnormal forms of Tau are found in different areas of the nervous system mostly in neuronal cells and, in some cases, in glial cells. The most common form of inclusions is called neurofibrillary tangles. They are found in AD, PD and in other tauopathies and consist of stacked paired helical filaments (PHFs) of hyper-phosphorylated Tau molecules [9]. In some diseases, Tau-mediated neuronal death can occur even in the absence of tangle formation [10], and it often aggregates with other teammates such as amyloid- $\beta$ or $\alpha$-synuclein $[11,12]$. 
Despite the extensive study of Tau aggregation over the last two decades, the exact causes of this process as well as its molecular mechanism are still not completely elucidated. PHF remains the most studied form of Tau aggregates. Among the endogenous factors that have been shown or suggested to favor Tau aggregation are post-transitional modifications (in particularly hyper-phosphorylation), mutations and the presence of zinc ions [13-16]. Interestingly, zinc ions were found to play a critical role in the development of several different NDs including $A D$ and PD. Zinc can bind not only to a number of prone-toaggregate proteins implicated in neurodegeneration (such as amyloid- $\beta$ [17], FUS/TLS [18], $\alpha$-synuclein [19], TDP-43 [20,21], etc.) and favor their aggregation, but also induce aggregation of stable proteins [22].

The potential impact of zinc ions on Tau aggregation/oligomerization was first discovered in 2009 [23]. It was shown that low micromolar concentrations of zinc are able to accelerate the fibrillization of the 244- to 372-aa fragment of human Tau40 (hTau40), which consists of the four MTBR binding repeats $R 1, R 2, R 3$ and $R 4$ [23]. Similar results have been recently obtained on this same fragment lacking R3 repeat [24]. Nevertheless, both studies were carried out in the presence of heparin, which induces Tau fibrillization into thioflavin $\mathrm{T}$ (ThT)positive PHFs. Recently, it was demonstrated that zinc not only accelerates aggregation of a pathological mutant $\Delta \mathrm{K} 280$ of $\mathrm{hTau} 40$ induced by Congo red in vitro, but also significantly increases its toxicity in neuronal cells [25]. This study also demonstrated the importance of Cys-291 and Cys-322 residues both for zinc binding and Tau aggregation [23,25]. While using non-physiological specific inductors of Tau aggregation such as heparin or Congo red is the most common way to induce PHFs structure and test inhibitors, it might not be the most pertinent model to study PHF formation itself, especially in the early stages where the process has already been hypothesized to be reversible [26]. Also, it should be noted that in these studies the pathological aggregation in the presence of zinc were demonstrated for Tau mutants and fragments that could have higher propensity to aggregate in comparison with wild type human full-length Tau (hTau40).

Here, we have investigated aggregation of fulllength human hTau40 isoform in the absence of heparin but in the presence of zinc ions under a wide range of temperature conditions using turbidimetry, isothermal titration calorimetry (ITC), dynamic light scattering (DLS) and transmission electron microscopy. We found that, contrary to its aggregation in the presence of heparin or with other inducers such as arachidonic acid or congo red [25,27,28], selfassembly of Tau is a reversible process that depends on temperature and is induced by zinc ions. Moreover, our findings point to an important role of low-affinity auxiliary zinc-binding sites in this process.

\section{Results}

\section{Zinc ions induce Tau oligomerization}

Turbidimetry was used to monitor the impact of zinc on Tau self-assembly in the absence of heparin at different temperatures. The absorbance $(\Delta \mathrm{A}$ $350 \mathrm{~nm}$ ) of $30 \mu \mathrm{M}$ Tau samples at different $\mathrm{ZnCl}_{2}$ concentrations (from 0 to $240 \mu \mathrm{M}$ ) in $50 \mathrm{mM}$ Tris buffer and $1 \mathrm{mM}$ TCEP at $\mathrm{pH} 7.5$ was measured over a wide range of temperatures from 15 to $95^{\circ} \mathrm{C}$ (Fig. 1a). In the absence of zinc ions as well as in the presence of low $\mathrm{ZnCl}_{2}$ concentrations $(15 \mu \mathrm{M})$, Tau protein did not demonstrate any propensity to aggregate. Increasing zinc concentration, starting from a $\mathrm{Zn} / \mathrm{Tau}$ molar ratio of 1 , led to a pronounced rise in the turbidity upon sample heating, which could indicate the formation of large oligomers of Tau. We found that Tau protein started to form oligomers at lower temperatures when higher zinc concentrations were used. Indeed, a 2-fold excess zinc induced Tau
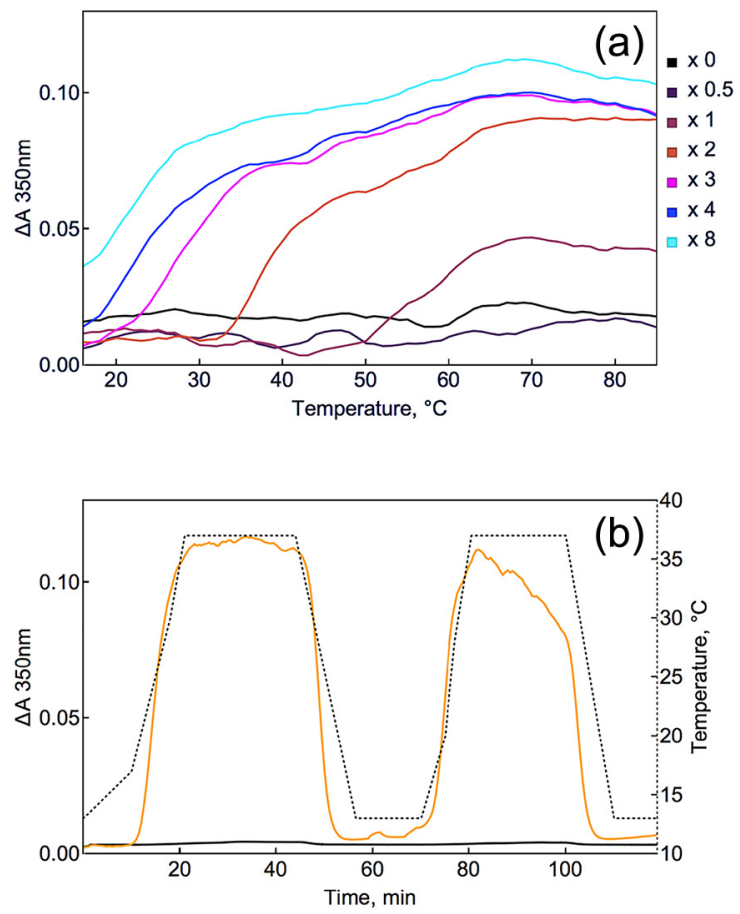

Fig. 1. (a) Temperature dependence of $30 \mu \mathrm{M}$ tau samples turbidity in the absence of $\mathrm{ZnCl}_{2}$ (black curve) and in the presence of 0.5-, 1-, 2-, 3-, 4- and 8-fold molar excess of $\mathrm{ZnCl}_{2}$ (see color legend on the right) in $50 \mathrm{mM}$ Tris buffer and $1 \mathrm{mM}$ TCEP at $\mathrm{pH} 7.5$ obtained using Prometheus NT.Plex instrument. (b) Reversible aggregation of tau $(30 \mu \mathrm{M})$ followed by turbidimetry in the presence of 4-fold molar excess of $\mathrm{ZnCl}_{2}$ (yellow curve); control tau sample in the absence of zinc (black curve); variation of temperature during experiment (black dotted line). Measurements were carried out using PerkinElmer spectrophotometer Lambda 800. 


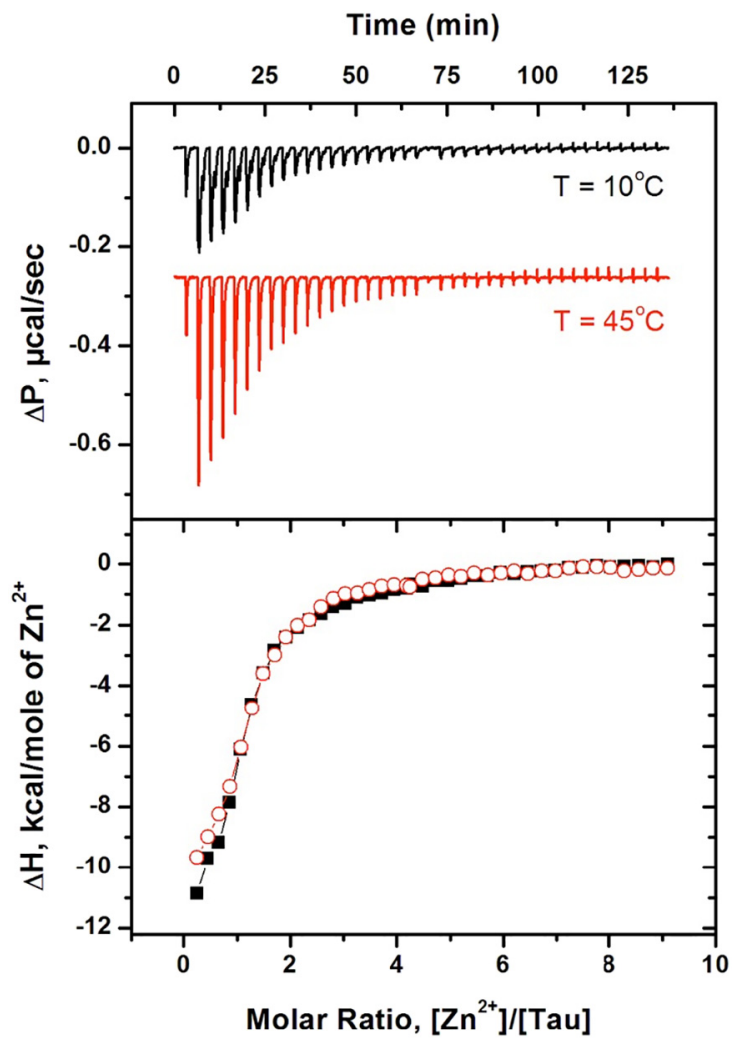

Fig. 2. Typical ITC titration curves (top panel) and binding isotherms (bottom panel) for zinc at $10^{\circ} \mathrm{C}$ (black) and at $45^{\circ} \mathrm{C}$ (red) interactions with $30 \mu \mathrm{M}$ of tau in $50 \mathrm{mM}$ Tris and $1 \mathrm{mM}$ TCEP at $\mathrm{pH} 7.5$.

assembly at near physiological temperatures, while in the presence of an 8-fold zinc excess, Tau was already partially assembled at $15{ }^{\circ} \mathrm{C}$. In parallel, to investigate the reversibility of this observed assembly, Tau sample was heated to $37^{\circ} \mathrm{C}$ and cooled down to $13^{\circ} \mathrm{C}$ in the presence of 4-fold zinc excess $\left(120 \mu \mathrm{M}\right.$ of $\mathrm{ZnCl}_{2}$ ). Each time, absorbance increased upon heating and dropped after cooling demonstrating near $100 \%$ reversibility of the oligomerization (Fig. 1b).

\section{Thermodynamics of Tau-zinc interaction}

To verify if Tau oligomerization is directly linked to zinc binding, we investigated Tau-zinc interaction using ITC at different temperatures. In the turbidimetry experiments, at low temperatures, we observed Tau aggregation only at high zinc concentrations (above $120 \mu \mathrm{M}$ ), but at high temperatures, Tau aggregation started at an equimolar zinc concentration. ITC titration of $30 \mu \mathrm{M}$ Tau solution by zinc at 10 and $45^{\circ} \mathrm{C}$ did not reveal significant differences in thermodynamic parameters of Tau-zinc interaction (Fig. 2), indicating that zinc binds to tau just as well at $10{ }^{\circ} \mathrm{C}$ than at $45^{\circ} \mathrm{C}$. In both cases, fitting the binding isotherms using a two-sets-of-sites model revealed the existence of one high-affinity site for zinc $\left(N=1.0 \pm 0.1 ; K_{\mathrm{a}}=2.0 \pm 0.5 \times 10^{6} \mathrm{M}^{-1}\right)$ and three low-affinity sites $\left(N=3.2 \pm 0.3 ; K_{\mathrm{a}}=5.9 \pm\right.$ $\left.1.7 \times 10^{4} \mathrm{M}^{-1}\right)$. The high-affinity site was enthalpy driven but entropy unfavorable $(\Delta H=-12.1 \pm$ $0.2 \mathrm{kcal} \mathrm{M}^{-1} ; \Delta S=-13.8 \mathrm{cal} \mathrm{M}^{-1} \mathrm{~K}^{-1}$ ) probably due to partial folding of initially unstructured Tau molecule. Meanwhile, the three low-affinity sites were both enthalpy and entropy favorable $(\Delta H=$ $-1.8 \pm 0.3 \mathrm{kcal} \mathrm{M}^{-1} ; \Delta S=15.4 \mathrm{cal} \mathrm{M}^{-1} \mathrm{~K}^{-1}$ ) pointing to the burying of hydrophobic surfaces upon interactions during Tau aggregation.

\section{Characterization of Tau oligomers}

To characterize the oligomers of Tau that form in the presence of zinc ions, we used DLS, transmission electron microscopy and ThT assay. DLS analysis of $30 \mu \mathrm{M}$ Tau solution showed that, in the absence of zinc, at $10^{\circ} \mathrm{C}$, Tau is mostly represented in solution as a species with a median hydrodynamic diameter of $9.6 \pm 1.4 \mathrm{~nm}$ by mass (Dv50; Table 1). Given that Tau is an intrinsically disordered protein, this size would correspond to the unfolded monomer [29]. Heating up to $40^{\circ} \mathrm{C}$ did not reveal significant conformational changes of the monomeric state of Tau (Table 1), in agreement with our turbidimetry data.

Table 1. Characteristics of Tau polymers in presence of different zinc concentrations by DLS

\begin{tabular}{lcllll}
\hline$T,{ }^{\circ} \mathrm{C}$ & {$\left[\mathrm{ZnCl}_{2}\right], \mu \mathrm{M}$} & \multicolumn{1}{c}{ Z-average, $\mathrm{nm}$} & \multicolumn{1}{c}{ Pdi } & Dv50, $\mathrm{nm}$ & Di50, $\mathrm{nm}$ \\
\hline 10 & 0 & $14.3 \pm 0.7$ & $0.31 \pm 0.02$ & $9.6 \pm 1.4$ & $13.7 \pm 1.1$ \\
40 & 0 & $20.0 \pm 2.1$ & $0.50 \pm 0.07$ & $12.2 \pm 0.6$ & $13,0 \pm 0.5$ \\
40 & 30 & $55.1 \pm 63.7$ & $0.30 \pm 0.13$ & $10.5 \pm 0.4$ & $12.2 \pm 0.2$ \\
40 & 60 & $193.3 \pm 46.1$ & $0.21 \pm 0.02$ & $11.4 \pm 0.2$ & $11.4 \pm 0.2$ \\
40 & 90 & $3158.0 \pm 1462.0$ & $0.99 \pm 0.02$ & $8.8 \pm 1.1$ & $8.9 \pm 1.2$ \\
40 & 120 & $99,000.0 \pm 20,000.0$ & $0 O R$ & OOR & OOR \\
$10^{\mathbf{a}}$ & 120 & $26.0 \pm 9.6$ & $0.27 \pm 0.08$ & $11.3 \pm 2.5$ & $13.2 \pm 0.3$ \\
40 & $90^{\mathbf{b}}$ & $55.2 \pm 30.2$ & $0.41 \pm 0.05$ & $11.3 \pm 0.3$ & $12.2 \pm 0.4$ \\
\hline
\end{tabular}

Z-average, mean intensity size (diameter) of sample; Pdi, polydispersity index; Dv50, volume-based diameter median; Di50, intensitybased diameter median in $\mathrm{nm}$; OOR, out of range.

a Sample cooled from 40 to $10^{\circ} \mathrm{C}$.

b 2 mM EDTA added. 
To investigate the impact of zinc ions on the oligomeric state of Tau, $30 \mu \mathrm{M}$ of Tau was titrated with up to a 4-fold molar excess of zinc at $40{ }^{\circ} \mathrm{C}$ and analyzed by DLS (Fig. $3 a-c)$. Within this range of zinc concentration, the main Tau species represented by mass had diameters ranging from 10 to $12 \mathrm{~nm}$. Furthermore, below the molar ratio $\mathrm{Zn} / \mathrm{Tau}$ of 2.3 , the polydispersity index (Pdi) remained low, while $Z$-average (mean particle size) gradually increased (Fig. 3c; Table 1). Above this $\mathrm{Zn} / \mathrm{Tau}$ ratio, $Z$ average started to increase together with the polydispersity indicating protein aggregation (Fig. 3c). Further increases in zinc concentration, up to excess of 4 , led to a complete disappearance of low size
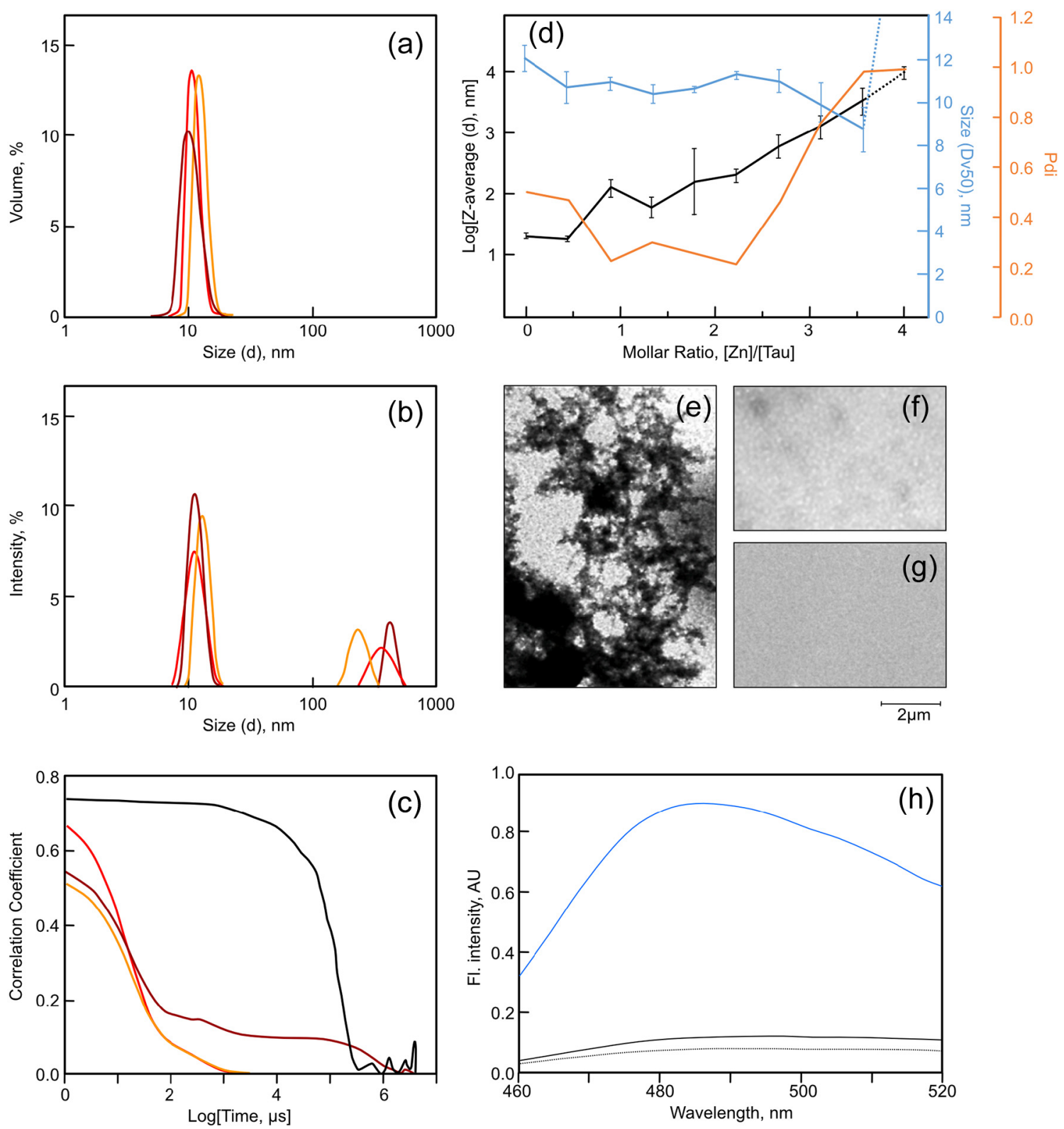

Fig. 3. Size distribution by volume (a) and by intensity (b) of $30 \mu \mathrm{M}$ tau in the absence of zinc (orange curve) and in the presence of 30 and $60 \mu \mathrm{M}$ zinc (in red and burgundy respectively) at $40{ }^{\circ} \mathrm{C}$. (c) Correlation coefficient versus time in $\mu$ s for Tau $30 \mu \mathrm{M}$ in the presence of 0,10,60 and $100 \mu \mathrm{M} \mathrm{ZnCl}$ (shown in colors orange, red, burgundy and black, respectively). (d) Median volume-based diameter (DV50) in nm (blue), Pdi (orange) and Z-average mean intensity size in (black) for different zinc/Tau molar ratio, with Tau $30 \mu \mathrm{M}$. (e) and (f) Representative electron micrographs of negatively stained Tau aggregates at $37{ }^{\circ} \mathrm{C}$ in the presence and in the absence of zinc ions respectively. (g) Representative electron micrographs of negatively stained sample of Tau in the presence cooled down from 37 to $10^{\circ} \mathrm{C}$. $(\mathrm{H})$ Fluorescence spectra of ThT at $60 \mu \mathrm{M}$ in buffer (dotted curve), in the presence of zinc-induced Tau aggregates (black curve) and in the presence of heparin-induced Tau aggregates (blue line) used as positive control. 
species (Fig. 3a, b) and the formation of oligomers too large to be properly monitored by DLS. Further investigation of formed structures by electron microscopy revealed the existence of large $(>10 \mu \mathrm{m})$ aggregates (Fig. 3e), which are in a good agreement with our DLS experiment. Indeed, as shown in the correlograms (Fig. 3d), as the concentration of zinc increases, the decay time increases, which indicates an increase in Tau size. Characterization of Tau oligomerization using ThT assay (Fig. $3 \mathrm{~g}$ ) did not reveal the presence of amyloid-like structures.

When the temperature was then decreased from 40 to $10^{\circ} \mathrm{C}$, we observed the reappearance of lowsize species with a Dv50 of $11.3 \pm 2.5 \mathrm{~nm}$ in the presence of $120 \mu \mathrm{M}$ zinc (Table 1), which indicates that the oligomerization induced by zinc at $40^{\circ} \mathrm{C}$ is reversible. Moreover, chelation of zinc ions with high excess of EDTA (2 mM) at $40^{\circ} \mathrm{C}$ also led to complete dissociation of aggregates, which returned to the same size distribution as it was before addition of zinc ions (Table 1). We have thus shown that reducing temperature can reverse the effect of zinc oligomerization of Tau and that removal of zinc at high temperatures can also reverse the oligomerization of Tau.

\section{Discussion}

Zinc, which plays an important role in many physiological processes, has been implicated in the molecular mechanisms of many NDs by favoring aggregation of proteins such as amyloid beta, FUS, TDP-43 [21,30]. Recent in vitro studies showed that zinc could also impact Tau aggregation into PHF, a hallmark of several tauopathies. Nevertheless, the conclusions from these studies were limited by the fact that the oligomerization of Tau into PHFs was induced by heparin.

Here, for the first time, we demonstrated that the presence of the zinc ions combined with increased temperature is sufficient to induce a fast oligomerization of Tau, and that it is reversible. Indeed, we have found that in the presence of equimolar zinc concentration, Tau oligomerizes at $50{ }^{\circ} \mathrm{C}$, while the increase of molar ratio of $\mathrm{Zn} / \mathrm{Tau}$ to 2 decreases the temperature of oligomerization to $32{ }^{\circ} \mathrm{C}$ (Fig. 1), thus potentially impacting physiological function of Tau. Similar interplay between ions and temperature of polymerization has already been observed in other important physiological systems such as $\mathrm{Mg}^{2+}$ / tubulin wherein tubulin polymerization depends both on temperature and $\mathrm{Mg}^{2+}$ concentration [31].

Characterization of $\mathrm{Zn} / \mathrm{Tau}$ binding by ITC at different temperatures allowed us to demonstrate that the thermodynamics of the interaction is the same whether Tau oligomerizes or not. Indeed, the binding isotherms registered at $10{ }^{\circ} \mathrm{C}$ (where oligomerization is only triggered by high zinc concentrations) and $45^{\circ} \mathrm{C}$ (where oligomerization starts at the beginning of titration) are almost identical. At both temperatures, ITC demonstrates the existence of two types of sites, one site with a high affinity $\left(K_{\mathrm{a}}=2.0 \pm\right.$ $\left.0.5 \times 10^{6} \mathrm{M}^{-1}\right)$ and three sites with a low affinity $\left(K_{\mathrm{a}}=5.9 \pm 1.7 \times 10^{4} \mathrm{M}^{-1}\right)$, which could have different functions. DLS showed that upon saturation of the high-affinity site, the hydrodynamic diameter of Tau species dropped from 12.2 to $10.8 \mathrm{~nm}$ and remained stable at $10.5 \pm 0.22 \mathrm{~nm}$ until a molar ratio $\mathrm{Zn} / \mathrm{Tau}$ of 2.3 (Fig. 3b). This suggests a partial folding of Tau with an increase in homogeneity as shown by the Pdi. This is also in agreement with the unfavorable entropy of zinc binding found by ITC for this site. This first high-affinity site likely corresponds to the one localized in the R2-R3 Tau region in which zinc is chelated by two Cys and two His amino acids, as previously proposed [23,25]. Such binding would be accompanied by the folding of Tau in R2-R3 as a hairpin, which is in good agreement with our DLS data that showed a compaction of the molecule. The three low-affinity sites may be responsible for the oligomerization process. Indeed, at $40^{\circ} \mathrm{C}$, we start to detect oligomerization by DLS at $\mathrm{Zn} / \mathrm{Tau}$ around 2 , when zinc starts to bind to these low-affinity sites, as it can be seen from ITC titration curve (Fig. 2). Interestingly, the hydrodynamic size of Tau monomer species continues to decrease (down to $8.9 \pm 1.2 \mathrm{~nm}$ ) as zinc binds to auxiliary sites, pointing to a further Tau folding (Fig. 3c). This leads us to propose that zinc binding to both types of sites induces a structural change that may have a consequence on Tau physiological functions.

It should be stressed that, contrary to heparininduced fibrillization of Tau in most PHF models, zincinduced oligomerization of Tau is a reversible process with fast kinetics. Indeed, chelation of zinc ions using EDTA, or a decrease of temperature instantly led to dissociation of oligomers. Contrary to ThT-positive irreversible aggregates formed in the presence of heparin, oligomers induced by zinc ions were not only reversible but also ThT-negative which indicates that the oligomers in the absence of heparin formed are not amyloid like. Most of the previous studies of Tau self-association in vitro were carried out in the presence of agents such as heparin or arachidonic acid $[27,28]$ and led to generation of irreversible structures. It has been hypothesized, and widely accepted, that irreversible Tau aggregation leading to PHFs found in neurons of patients affected with ND would be preceded by a stage of reversible Tau multimers formation [26]. In our study, for the first time, we were able to reproduce a reversible oligomerization of Tau proteins in the presence of $\mathrm{Zn}$ without any additional non-physiological additives. Thus, our experimental conditions could constitute a useful model to study Tau multimerization in vitro.

The reversibility of Tau structures observed in our experiments also allows us to hypothesize that 
oligomers could be induced in cells by zinc signals under physiological conditions and play a role in Tau regulation, modulating its numerous functions [1]. For example, zinc was found to be involved in regulation of Tau interaction with DNA [32]. More generally, zinc is abundantly present in the brain where it plays an important role in axonal and synaptic transmission [33,34]. It is highly concentrated in the synaptic vesicles of several types of neurons [35]. The majority of zinc ions in cell are bound to metalloproteins, which have metal binding affinities in the $\mathrm{nM}$ and even in $\mathrm{pM}$ range. The physiological concentration of free $\mathrm{Zn}^{2+}$ in cells is considered to be between around 1 and $10 \mathrm{nM}$ $[36,37]$, which locally would be sufficient to bind to Tau and modulate its activity. Thus, the observed binding constants that we determined are consistent with the possible physiological role of $\mathrm{Zn}$ in Tau regulation.

\section{Conclusion}

In conclusion, we demonstrated for the first time that zinc can induce a reversible polymerization of Tau. This process could have a significant function in the brain and nervous system where zinc is known to be abundant. Our data, which demonstrate that zinc favors Tau self-assembly at near physiological temperatures, support the idea that zinc could be implicated both in the pathological aggregation of Tau and in tau regulation. As summarized in Fig. 4, while the right part depicts the irreversible cascade leading to the formation of neurofibrillary tangles, the left part depicts a pathway that would ultimately lead to the formation reversible granular aggregates of Tau, which could have a sequestration purpose. Since tau phosphorylation is known to have physiological and pathological consequences [38], impact of tau phosphorylation on this pathway will need to be addressed. We have also shown the existence of two types of sites (one main and three auxiliary ones) that gradually modulate Tau structure enabling zinc to be a subtle modulator of Tau activity. In order to understand how zinc ions can impact physiological Tau oligomerization, it will be important to identify the specific amino acids responsible for binding of those auxiliary zinc ions and the structure of such oligomers. Likewise, confirming that this reversible oligomerization of Tau is part of a concurrent pathway will have important consequences in ND research.

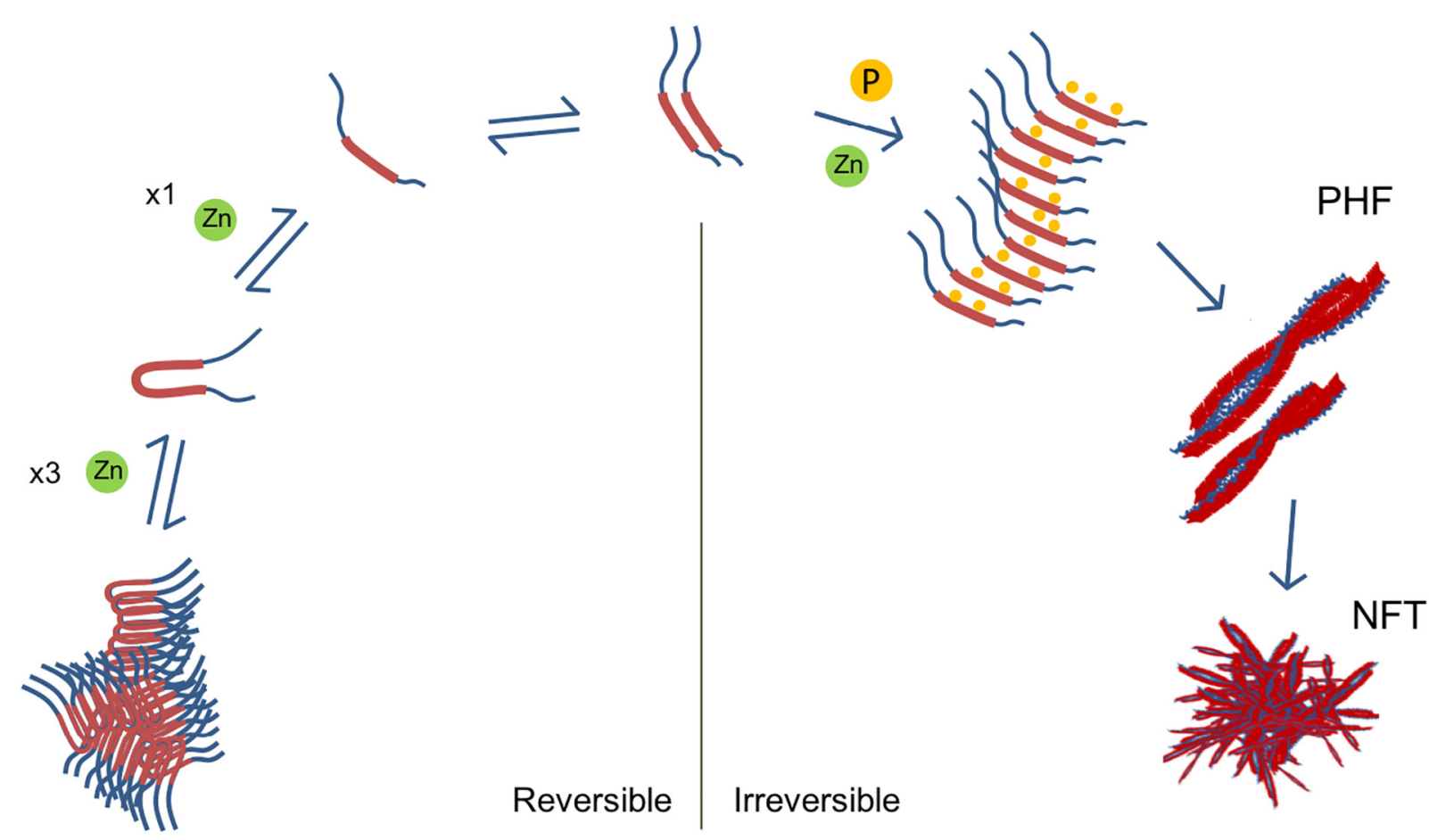

Fig. 4. Model. The right part of the scheme corresponds to the pathway leading to PHFs and neurofibrillary tangle formation. This pathway is globally irreversible and favored by post-translational modifications such as (hyper) phosphorylation of Tau and $\mathrm{Zn}^{2+}$. The left part of the scheme summarizes our findings: in the presence of low $\mathrm{Zn}^{2+}$ concentrations, Tau undergoes compaction; in the presence of high $\mathrm{Zn}^{2+}$ concentrations, it leads to the formation of granular Tau oligomers. This pathway is fully reversible. 


\section{Materials and Methods}

\section{Protein purification}

Human Tau (hTau40) was expressed in Escherichia coli and purified as described previously [39]. Tau could thus be directly resuspended before use in the appropriate buffer. Protein was passed through the Zeba Spin Desalting Columns (Thermo Scientific) to equilibration in Tris $50 \mathrm{mM}$ and TCEP $1 \mathrm{mM}(\mathrm{pH} 7.5)$ buffer before being used for experiments. TCEP was used to maintain Tau in a reduced state. Its concentration was measured at $280 \mathrm{~nm}$ using extinction a coefficient of $7700 \mathrm{M}^{-1} \mathrm{~cm}^{-1}$ [31].

\section{ITC}

Binding of zinc to Tau was analyzed by ITC using MicroCal iTC200 as described previously $[40,41]$. Experiments were performed at 10 and $45^{\circ} \mathrm{C}$ and in $50 \mathrm{mM}$ Tris buffer in the presence of $1 \mathrm{mM}$ TCEP at $\mathrm{pH}$ 7.5. Tau concentration in the calorimetric cell was $30 \mu \mathrm{M}$, whereas the $\mathrm{ZnCl}_{2}$ concentration in the syringe was $600 \mu \mathrm{M}$. Tau was titrated by repeated injections of $2 \mu \mathrm{L}$ aliquots of zinc solution. At the end of titration, the cell contained the mixture of zinc and Tau with the molar ratio equal to $4: 1$. The syringe was refilled with the same zinc solution without cell refilling, and the titration was continued until the final ratio of 8:1. Each resulting titration peak was integrated and plotted as a function of the Tau/ tubulin molar ratio. The baseline was measured by injecting $\mathrm{ZnCl}_{2}$ into the protein-free buffer solution. Data were analyzed using Origin software and were fitted with a "two set of sites" model via a non-linear least squares minimization method and led to the determination of affinity constants $\left(K_{\mathrm{a}}\right)$ and enthalpy changes $(\Delta H)$. Thermodynamic values are an average of at least three different experiments.

\section{Turbidimetry}

Tau polymerization was monitored by turbidimetry (A $350 \mathrm{~nm}$ ) using a PerkinElmer spectrophotometer Lambda 800 (PerkinElmer) and a Prometheus NT. Plex (Nanotemper) instrument equipped with absorbance module with a heating rate of $1 \mathrm{~K} / \mathrm{min}$. Purified Tau $(30 \mu \mathrm{M})$ was equilibrated in $50 \mathrm{mM}$ Tris and $1 \mathrm{mM}$ TCEP $(\mathrm{pH} 7.5)$ buffer in the absence or in the presence of zinc and incubated at $5{ }^{\circ} \mathrm{C}$. Polymerization was initiated by temperature increase to $40{ }^{\circ} \mathrm{C}$ and reversed by cooling sample to $13^{\circ} \mathrm{C}$.

\section{DLS}

DLS experiments were carried out to determine the hydrodynamic diameter of Tau in the presence and absence of zinc using a Zetasizer Nano ZS
(Malvern Instruments) with a scattering angle of $173^{\circ}$. Particles in solution are in constant random motion and the intensity of their scattered light fluctuates with time. To determine the hydrodynamic diameter $\left(D_{\mathrm{h}}\right)$, the provided software uses the StokesEinstein relation to obtain the intensity averaged size distribution from the raw correlation data. The correlogram displays much information about the sample. The time at which the correlation starts to decay is an indication of the mean size of the sample. Smaller samples fluctuate quicker than larger samples in solution. The steeper the exponential decay, the more monodisperse (single population) the sample, and the more the decay is extended the greater the sample polydispersity (several populations) which is indicated by the Pdi. To analyze Tau, DLS was performed at temperatures ranging from 10 to $40^{\circ} \mathrm{C}$. For each assay, three measurements were performed; each one consisting in 10-15 runs of $10 \mathrm{~s}$. Tau was analyzed at $30 \mu \mathrm{M}$ in $50 \mathrm{mM}$ Tris and $1 \mathrm{mM}$ TCEP ( $\mathrm{pH}$ 7.5) following centrifugation for $15 \mathrm{~min}$ at $14,000 \mathrm{rpm}$ at $6{ }^{\circ} \mathrm{C}$. The overall thermostability and aggregation of Tau was tested in the absence and presence of $10-120 \mu \mathrm{M}$ of $\mathrm{ZnCl}_{2}$. To determine the hydrodynamic diameter $\left(D_{\mathrm{h}}\right)$, the viscosity and refractive index values of the dispersion medium were used in the Stokes-Einstein equation. In our case, we used a viscosity of $1.3198 \mathrm{cP}$ and a refractive index of 1.331 (at $10^{\circ} \mathrm{C}$ ). Results were displayed as volume and intensity size distributions including the overall mean size (Z-average) using the Zetasizer software 7.12 with a detailed highresolution spectrum of 300 classes. All experiments were performed at least in triplicates.

\section{Fluorescence ThT assay}

ThT assay, which is a common marker of amyloid fibrils, was performed as described before [42] with some modifications. Briefly, samples of $30 \mu \mathrm{M}$ Tau alone or Tau with 8-fold excess of $\mathrm{ZnCl}_{2}$ were mixed with ThT. Fluorescence spectra were acquired in 0.2 (excitation direction) $\times 1 \mathrm{~cm}$ (emission) cells (Hellma) thermostated at $37^{\circ} \mathrm{C} \pm 0.5^{\circ} \mathrm{C}$ without preincubation. Excitation was at $440 \mathrm{~nm}$ and emission spectra were recorded from 460 to $520 \mathrm{~nm}$ with slit widths of $15 / 15 \mathrm{~nm}$ using a PerkinEImer LS 55 fluorescence spectrometer operating at a PM of $800 \mathrm{~V}$.

\section{Transmission electron microscopy}

Four microliters of incubated Tau samples $(30 \mu \mathrm{M})$ was placed on carbon-coated copper grids (300 mesh) for $1 \mathrm{~min}$. After blotting, grids were washed with distilled water and blotted again, and negatively stained for $30 \mathrm{~s}$ with $2 \%$ (wt/vol) uranyl acetate as previously described [21]. The grids were then dried and observed with a JEOL 2200FS transmission electron microscope (Tokyo, Japan) operating at 
$200 \mathrm{kV}$. Images were recorded using a $4 \mathrm{k} \times 4 \mathrm{k}$ slowscan CCD camera (Gatan, Inc., Pleasanton, USA).

\section{CRediT authorship contribution state- ment}

Andrei Yu. Roman: Formal analysis. François Devred: Conceptualization, Supervision, Project administration, Funding acquisition, Writing - review \& editing. Deborah Byrne: Methodology, Writing review \& editing. Romain La Rocca: Formal analysis. Natalia N. Ninkina: Writing - review \& editing. Vincent Peyrot: Writing - review \& editing. Philipp 0. Tsvetkov: Conceptualization, Data curation, Formal analysis, Supervision, Writing - original draft, Writing review \& editing.

\section{Acknowledgments}

Microcalorimetry and turbidimetry experiments were performed in Timone Microcalorimetry Platform, PINT, Marseille, France. This study is a part of $\mathrm{PhD}$ research of A.Y.R. funded by "Doctoral scholarships Metchnikov" from the French embassy in Moscow. We are grateful to Prof. Rosalind John for reading the manuscript and for valuable comments.

Ethics Approval and Consent to Participate: Not applicable.

Consent for Publication: Not applicable.

Availability of Data and Materials: All data generated or analyzed during this study are included in this published article.

Funding: A.Y.R. was funded by a Metchnikov grant form the French Embassy of Moscow.

Authors Contributions: A.Y.R. purified the recombinant protein and performed all experiments; F.D. supervised the experiments and wrote the manuscript; D.B. performed DLS data analysis and wrote the manuscript; R.L.R. performed experiments; V.P. and N.N.N. supervised the research; P.O.T. designed the research, performed data analysis and wrote the manuscript. All authors read and approved the final manuscript.

Competing Interests: The authors declare that they have no competing interests.

Received 5 June 2018; Received in revised form 14 December 2018; Accepted 14 December 2018 Available online $\mathrm{xxxx}$

Keywords:

Tau;

zinc;

aggregation self-assembly
Abbreviations used:

MT, microtubule; ND, neurodegenerative diseases; AD,

Alzheimer's disease; PD, Parkinson's disease; PHF, paired helical filaments; ThT, thioflavin T; ITC, isothermal titration calorimetry; DLS, dynamic light scattering.

\section{References}

[1] I. Sotiropoulos, M.C. Galas, J.M. Silva, E. Skoulakis, S. Wegmann, M.B. Maina, et al., Atypical, non-standard functions of the microtubule associated Tau protein, Acta Neuropathol. Commun. 5 (2017) 91.

[2] Y. Wang, E. Mandelkow, Tau in physiology and pathology, Nat. Rev. Neurosci. 17 (2016) 5-21.

[3] M. Goedert, M.G. Spillantini, Propagation of Tau aggregates, Mol. Brain 10 (2017) 18.

[4] M. Goedert, A. Klug, R.A. Crowther, Tau protein, the paired helical filament and Alzheimer's disease, J. Alzheimers Dis. 9 (2006) 195-207.

[5] S.M. Ward, D.S. Himmelstein, J.K. Lancia, L.I. Binder, Tau oligomers and tau toxicity in neurodegenerative disease, Biochem. Soc. Trans. 40 (2012) 667-671.

[6] T.A. Shelkovnikova, A.A. Kulikova, F.O. Tsvetkov, O. Peters, S.O. Bachurin, V.L. Bukhman, et al., Proteinopathies-forms of neurodegenerative disorders with protein aggregation-based pathology, Mol. Biol. (Mosk) 46 (2012) 402-415.

[7] A. Mudher, M. Colin, S. Dujardin, M. Medina, I. Dewachter, S.M. Alavi Naini, et al., What is the evidence that tau pathology spreads through prion-like propagation? Acta Neuropathol. Commun. 5 (2017) 99.

[8] F. Kametani, M. Hasegawa, Reconsideration of amyloid hypothesis and tau hypothesis in Alzheimer's disease, Front. Neurosci. 12 (2018) 25.

[9] A. Ochalek, B. Mihalik, H.X. Avci, A. Chandrasekaran, A. Teglasi, I. Bock, et al., Neurons derived from sporadic Alzheimer's disease iPSCs reveal elevated TAU hyperphosphorylation, increased amyloid levels, and GSK3B activation, Alzheimers Res. Ther. 9 (2017) 90.

[10] T.F. Gendron, L. Petrucelli, The role of tau in neurodegeneration, Mol. Neurodegener. 4 (2009) 13.

[11] J.E. Gerson, K.M. Farmer, N. Henson, D.L. Castillo-Carranza, M. Carretero Murillo, U. Sengupta, et al., Tau oligomers mediate alpha-synuclein toxicity and can be targeted by immunotherapy, Mol. Neurodegener. 13 (2018) 13.

[12] S. Moussaud, D.R. Jones, E.L. Moussaud-Lamodiere, M. Delenclos, O.A. Ross, P.J. McLean, Alpha-synuclein and tau: teammates in neurodegeneration? Mol. Neurodegener. 9 (2014) 43.

[13] A.C. Kim, S. Lim, Y.K. Kim, Metal ion effects on Abeta and tau aggregation, Int. J. Mol. Sci. 19 (2018).

[14] T. Kimura, G. Sharma, K. Ishiguro, S.I. Hisanaga, Phosphotau bar code: analysis of phosphoisotypes of tau and its application to tauopathy, Front. Neurosci. 12 (2018) 44.

[15] M. Inoue, S. Kaida, S. Nakano, C. Annoni, E. Nakata, T. Konno, et al., Phosphorylation regulates fibrillation of an aggregation core peptide in the second repeat of microtubulebinding domain of human tau, Bioorg. Med. Chem. 22 (2014) $6471-6480$

[16] S.L. Forrest, J.J. Kril, C.H. Stevens, J.B. Kwok, M. Hallupp, W.S. Kim, et al., Retiring the term FTDP-17 as MAPT mutations are genetic forms of sporadic frontotemporal tauopathies, Brain 141 (2018) 521-534. 
[17] S.A. Kozin, A.A. Kulikova, A.N. Istrate, P.O. Tsvetkov, S.S. Zhokhov, Y.V. Mezentsev, et al., The English (H6R) familial Alzheimer's disease mutation facilitates zinc-induced dimerization of the amyloid-beta metal-binding domain, Metallomics 7 (2015) 422-425.

[18] A.D. Efimova, R.K. Ovchinnikov, A.Y. Roman, A.V. Maltsev, V.V. Grigoriev, E.A. Kovrazhkina, et al., The FUS protein: physiological functions and a role in amyotrophic lateral sclerosis, Mol. Biol. (Mosk) 51 (2017) 387-399.

[19] A.A. Valiente-Gabioud, V. Torres-Monserrat, L. MolinaRubino, A. Binolfi, C. Griesinger, C.O. Fernandez, Structural basis behind the interaction of $\mathrm{Zn}(2)(+)$ with the protein alpha-synuclein and the Abeta peptide: a comparative analysis, J. Inorg. Biochem. 117 (2012) 334-341.

[20] A. Caragounis, K.A. Price, C.P. Soon, G. Filiz, C.L. Masters, Q.X. Li, et al., Zinc induces depletion and aggregation of endogenous TDP-43, Free Radic. Biol. Med. 48 (2010) 1152-1161.

[21] C. Garnier, F. Devred, D. Byrne, R. Puppo, A.Y. Roman, S. Malesinski, et al., Zinc binding to RNA recognition motif of TDP-43 induces the formation of amyloid-like aggregates, Sci. Rep. 7 (2017) 6812.

[22] P.O. Tsvetkov, A.Y. Roman, V.E. Baksheeva, A.A. Nazipova, M.P. Shevelyova, V.I. Vladimirov, et al., Functional status of neuronal calcium sensor-1 is modulated by zinc binding, Front. Mol. Neurosci. 11 (2018).

[23] Z.Y. Mo, Y.Z. Zhu, H.L. Zhu, J.B. Fan, J. Chen, Y. Liang, Low micromolar zinc accelerates the fibrillization of human tau via bridging of Cys-291 and Cys-322, J. Biol. Chem. 284 (2009) 34648-34657.

[24] A.C. Jiji, A. Arshad, S.R. Dhanya, P.S. Shabana, C.K. Mehjubin, V. Vijayan, Zn(2+) interrupts R4-R3 association leading to accelerated aggregation of tau protein, Chemistry 23 (2017) 16976-16979.

[25] J.Y. Hu, D.L. Zhang, X.L. Liu, X.S. Li, X.Q. Cheng, J. Chen, et al., Pathological concentration of zinc dramatically accelerates abnormal aggregation of full-length human Tau and thereby significantly increases Tau toxicity in neuronal cells, Biochim. Biophys. Acta 2017 (1863) 414-427.

[26] T. Arendt, J. Stieler, A.M. Strijkstra, R.A. Hut, J. Rudiger, E.A. Van der Zee, et al., Reversible paired helical filament-like phosphorylation of tau is an adaptive process associated with neuronal plasticity in hibernating animals, J. Neurosci. 23 (2003) 6972-6981.

[27] M.E. King, T.C. Gamblin, J. Kuret, L.I. Binder, Differential assembly of human tau isoforms in the presence of arachidonic acid, J. Neurochem. 74 (2000) 1749-1757.

[28] Y. Mutreja, T.C. Gamblin, Optimization of in vitro conditions to study the arachidonic acid induction of $4 \mathrm{R}$ isoforms of the microtubule-associated protein tau, Methods Cell Biol. 141 (2017) 65-88.

[29] Z. Yu, J.C. Reid, Y.P. Yang, Utilizing dynamic light scattering as a process analytical technology for protein folding and aggregation monitoring in vaccine manufacturing, J. Pharm. Sci. 102 (2013) 4284-4290.

[30] H. Kozlowski, M. Luczkowski, M. Remelli, D. Valensin, Copper, zinc and iron in neurodegenerative diseases (Alzheimer's, Parkinson's and prion diseases), Coord. Chem. Rev. 256 (2012) 2129-2141.

[31] F. Devred, P. Barbier, S. Douillard, O. Monasterio, J.M. Andreu, V. Peyrot, Tau induces ring and microtubule formation from alphabeta-tubulin dimers under nonassembly conditions, Biochemistry 43 (2004) 10520-10531.

[32] K. Asadollahi, G. Riazi, A. Rabbani Chadegani, S. Rafiee, DNA-binding mode transition of tau in the presence of zinc ions, J. Biomol. Struct. Dyn. (2017) 1-9.

[33] A. Takeda, Zinc homeostasis and functions of zinc in the brain, Biometals 14 (2001) 343-351.

[34] S.D. Gower-Winter, C.W. Levenson, Zinc in the central nervous system: from molecules to behavior, Biofactors 38 (2012) 186-193.

[35] P. Paoletti, A.M. Vergnano, B. Barbour, M. Casado, Zinc at glutamatergic synapses, Neuroscience 158 (2009) 126-136.

[36] D.J. Eide, Zinc transporters and the cellular trafficking of zinc, Biochim. Biophys. Acta 2006 (1763) 711-722.

[37] X.Y. Sun, Y.P. Wei, Y. Xiong, X.C. Wang, A.J. Xie, X.L. Wang, et al., Synaptic released zinc promotes tau hyperphosphorylation by inhibition of protein phosphatase 2A (PP2A), J. Biol. Chem. 287 (2012) 11174-11182.

[38] W.H. Stoothoff, G.V. Johnson, Tau phosphorylation: physiological and pathological consequences, Biochim. Biophys. Acta 2005 (1739) 280-297.

[39] T. De Bessa, G. Breuzard, D. Allegro, F. Devred, V. Peyrot, P. Barbier, Tau interaction with tubulin and microtubules: from purified proteins to cells, Methods Mol. Biol. 1523 (2017) 61-85.

[40] P.O. Tsvetkov, P. Barbier, G. Breuzard, V. Peyrot, F. Devred, Microtubule-associated proteins and tubulin interaction by isothermal titration calorimetry, Methods Cell Biol. 115 (2013) 283-302.

[41] P.O. Tsvetkov, A.A. Makarov, S. Malesinski, V. Peyrot, F. Devred, New insights into tau-microtubules interaction revealed by isothermal titration calorimetry, Biochimie 94 (2012) 916-919.

[42] D. Sui, M. Liu, M.H. Kuo, In vitro aggregation assays using hyperphosphorylated tau protein, J. Vis. Exp. 95 (2015), e51537. https://doi.org/10.3791/51537. 